\title{
Management of knee osteoarthritis by combined stromal vascular fraction cell therapy, platelet-rich plasma, and musculoskeletal exercises:
}

\section{a case series}

\author{
This article was published in the following Dove Press journal: \\ Journal of Pain Research \\ 9 November 2015 \\ Number of times this article has been viewed
}

\section{Nathan Gibbs' \\ Rod Diamond ${ }^{2}$ \\ Eric O Sekyere ${ }^{3}$ \\ Wayne D Thomas ${ }^{4}$}

'South Sydney Sports Medicine, Kensington, ${ }^{2}$ Diamond Health Care, Kensington, ${ }^{3}$ Endeavour College of Natural Health, Sydney, ${ }^{4}$ CellInnovations Pty Ltd, Liverpool, NSW, Australia
Correspondence: Nathan Gibbs South Sydney Sports Medicine, III Anzac Parade, Kensington, NSW 2033, Australia $\mathrm{Tel}+6 \mid 296635999$

Fax +61296633830

Email nathangibbs@bigpond.com
Introduction: Knee osteoarthritis is associated with persistent joint pain, stiffness, joint deformities, ligament damage, and surrounding muscle atrophy. The complexity of the disease makes treatment difficult. There are no therapeutic drugs available to halt the disease progression, leaving patients dependent on pain medication, anti-inflammatory drugs, or invasive joint replacement surgery.

Case presentations: Four patients with a history of unresolved symptomatic knee osteoarthritis were investigated for the therapeutic outcome of combining an exercise rehabilitation program with intra-articular injections of autologous StroMed (ie, stromal vascular fraction cells concentrated by ultrasonic cavitation from lipoaspirate) and platelet-rich plasma (PRP). The Knee Injury and Osteoarthritis Outcome Score questionnaire (KOOS) was administered along with physical function tests over a 12-month period. The first patient achieved a maximum therapeutic outcome of 100 in all five KOOS subscales (left knee), and 100 for four subscales (right knee). The second patient scored 100 in all five KOOS subscales (left knee), and greater than 84 in all subscales (right knee). Treatment of the third patient resulted in improved outcomes in both knees of $>93$ for four KOOS subscales, and 60 for the Function in Sport and Recreation subscale. The fourth patient improved to 100 in all five KOOS subscales. In all patients, the physical function "Get-up and Go" test and "Stair Climbing Test" returned to normal (a value of zero).

Conclusion: This case series indicates that improved outcomes may be obtained when autologous stromal vascular fraction (StroMed) cell therapy is combined with traditional exercise practices and PRP for osteoarthritis. Of the seven joints treated: all patients' scores of pain improved to $>96$; and quality of life scores to $>93$. Functional performance measures of mobility returned to normal. This simple treatment appears to be extremely effective for osteoarthritis disorders that have no drug treatment to halt disease progression.

Keywords: autologous stromal cell concentrate, SVF, StroMed, mesenchymal stem cells, pain, joint

\section{Introduction}

Regenerative medicine has continued to gain clinical significance by research evidence from both animal and human studies, clinical trials, and clinical practice. The therapeutic rationale for regenerative medicine is that cell-based therapies have the potential to return the patient to health with respect to that condition, without further treatment. Most medications for chronic conditions are unable to do this. It is now well recognized that damaged mammalian tissues do repair or self-regenerate and that - during gestation - injured or damaged neonatal tissues can be wholly recreated. ${ }^{1}$ 
While this inherent capacity decreases with age and chronic injury conditions, evidence for regenerative or self-repair pathways in adult mammals remains incontrovertible with adult stem cell populations now isolated successfully from a variety of tissues in the body, including bone marrow, adipose tissue, muscle, dermis, liver, neural, olfactory, and tendon. Mesenchymal stem cells (MSCs) are the focus for the development of many cell-based therapies for a diverse array of diseases, and are a subset of nonhematopoietic adult stem cells that originate from the mesoderm and exist in almost all adult tissues. ${ }^{2}$ The relative abundance of MSCs throughout the body is understandable, if most MSCs are of perivascular origin. ${ }^{3}$

MSCs have been demonstrated to secrete a broad range of bioactive or trophic factors that are essential in the cellular microenvironment for survival, protection, immune modulation, and differentiation effects. ${ }^{4}$ Trophic factors include $\mathrm{C}$ granulocyte; interleukins-6,-7,-8, and -11; macrophage colony-stimulating factors; tumor necrosis factor-alpha; hepatocyte growth factor; granulocytestimulating factors; macrophage-stimulating factors 11 ; vascular endothelial growth factor; nerve growth factor; brain-derived neurotrophic factor; adipokines; and several others. ${ }^{4}$ Experimental evidence have also demonstrated that MSCs have the capabilities of differentiating into adipocytic, chondrocytic, and osteocytic lineages on suitable stimulation. $^{5}$ These cellular properties may make MSCs suitable for cartilage and bone repair. Indeed, clinically, bone marrow- and adipose-derived tissue stem cell populations have been used with degrees of success in fracture repair, ${ }^{6}$ osteonecrotic therapy, ${ }^{7}$ cartilage repair, ${ }^{8}$ spinal fusion, ${ }^{9}$ and arthritis. ${ }^{10}$

Adipose tissue is a rich source of MSCs, and they are identified as adipose-derived stromal stem cells (ASCs). On separation of the fat cells from the adipose tissue, the remaining concentrated noncultured heterogeneous population of mononuclear cells is termed stromal vascular fraction (SVF) cells. This term is more than 4 decades old and, as a source of stem cells, was first described by Zuk et al, ${ }^{11}$ who identified MSC-like cells in SVF. The concentration of native ASCs, defined as fibroblastoid colony-forming units, has been observed to be $\sim 0.06 \%$ to $4 \%$ in SVF, providing the therapeutic rationale for use of this cell population. ${ }^{12-14}$ SVF also comprises other cell types including preadipocytes, endothelial progenitor cells, adipocytes, fibroblasts, pericytes, T-regulatory cells, monocytes, lymphocytes, vascular smooth muscle cells, and M2 macrophages. ${ }^{15}$ The heterogeneous content of SVF has been reported to assist the modulation and vascularization of the targeted tissue, contribute to the reduction of inflammation, and favor the re-equilibration of tolerogenic mechanisms. ${ }^{16,17}$

SVF as a cell source for MSC regenerative therapy has many advantages; it contains abundant MSCs, is easily extracted from the patient's own autologous adipose tissue, the tissue supply is plentiful, is harvested with a minimally invasive procedure (by mini-liposuction aspiration), and is able to be replenished. Several methods are available for the separation of SVF from lipoaspirate including ultrasonic cavitation and collagenase. The SVF product obtained by ultrasonic cavitation has been termed "StroMed". The viability and differentiation capabilities of the cells in StroMed have been confirmed by the adherence, proliferation, and differentiation of cells into adipocytic, chondrocytic, and osteocytic lineages (unpublished data). Cell therapy treatment using autologous SVF and undertaken by a medical practitioner is a procedure permitted by the Therapeutic Goods Administration of Australia (Excluded Goods Order 2011). Informed consent was obtained from each patient, and the study protocol conformed to the principles of the 1975 Declaration of Helsinki.

Osteoarthritis is a common chronic musculoskeletal disorder of the joints affecting the cartilage, ligaments, joint lining, and surrounding bones. It is caused by the progressive deterioration in the integrity of the joint structures and is associated with inflammation of the supporting cartilaginous structures within the joint capsule, and stiffness, pain, and reduction in mobility and normal function. Osteoarthritis can result in a reduced quality of life (QOL) and higher mortality rates. It can affect both the working age population and the elderly and is a leading cause of disability in the elderly. Age, sex, obesity, excessive joint loading, injuries to the joint, metabolic dysfunction, and genetic and environmental factors are known risk factors. ${ }^{18}$ The incidence of osteoarthritis is increasing rapidly in parallel with the rising aging and obese populations, and is placing a burden on health care budgets. Despite its debilitating effects there are, as yet, no reliable or effective treatments that can prevent osteoarthritis or halt the disease progression.

Clinical observation and magnetic resonance imaging (MRI) scans of the arthritic knee show clear joint deformities, poor bone structure and alignment, cartilage wear, ligament damage, muscle atrophy, and meniscal tears. The physical and clinical histories of osteoarthritis disorders point to a multitissue, multicell involvement in the etiology 
of the syndrome. This suggests that a multitreatment approach that recognizes the range of the underlying causes and symptoms in the osteoarthritic syndrome is critical to treatment. Current treatment options are based on combinations of patient education; physical exercise; nonsteroidal anti-inflammatory medication to relieve pain and inflammation; weight control; and, eventually, invasive total joint replacement surgery.

Moderate exercise has been shown to be effective in improving physical function, muscle strength, and flexibility, and in reducing joint pain and stiffness. In addition, platelet-rich plasma (PRP) preparations are increasingly being used in different clinical scenarios as a growth factor pool to treat a range of degenerative diseases (including cartilage lesions and knee osteoarthritis), although efficacy is debatable. ${ }^{19}$ Therefore, given the multietiological nature of the osteoarthritis syndrome and its effect on multiple tissues and structures within the entire joint, the combination of a StroMed cell-based and PRP therapy, supplemented with moderate exercise, holds promise for cartilage regeneration and repair, and, importantly pain and inflammation relief, leading to a reduction in medications.

\section{Case presentations}

A single dose of freshly prepared, noncultured SVF cells (average range $5 \times 10^{7}$ to $1.15 \times 10^{7}$, with $>85 \%$ viability) was prepared from the patient's own adipose tissue and injected along with $3 \mathrm{~mL}$ of autologous PRP into the osteoarthritic knee. Case study patients also received monthly injections of PRP for 4 months after the initial injection, followed by the occasional PRP injection. Three or six months posttreatment, patients were enrolled in a moderate exercise program for a total of 4 months.

SVF cells and PRP were prepared as follows: the lipoaspirate procedure was performed under local anesthetic only. Three millimeter puncture holes were created subcutaneously on either side of the periumbilical region (to avoid previous surgical incisions). This region was injected with $2 \mathrm{~mL}$ of $2 \%$ lignocaine with adrenaline after cleaning with betadine antiseptic. Then, $150 \mathrm{~mL}$ tumescent fluid (400 mg lignocaine, $1 \mathrm{mg}$ adrenaline, and $840 \mathrm{mg}$ sodium bicarbonate per $1,000 \mathrm{~mL}$ normal saline) was injected subcutaneously into each of the puncture hole sites via a $21 \mathrm{G} 10 \mathrm{~cm}$ needle. This process resulted in a relatively painless and bloodless liposuction using a $10 \mathrm{~cm}$ long and $3 \mathrm{~mm}$ wide cannula connected to a $25 \mathrm{~mL}$ syringe. Four $25 \mathrm{cc}$ syringes of lipoaspirate in total were collected. The puncture hole sites were not sutured but dressed with a compression bandage to be worn for the first 24 hours. Postprocedure pain was minimal and required only mild analgesics such as paracetamol. Routine prophylactic antibiotics were not given.

The lipoaspirate was then extracted from the four syringes and processed using the Cell-Innovations Pty Ltd standard ultrasonic cavitation proprietary protocol under sterile conditions to obtain "StroMed". ${ }^{20}$ The cell solution was filtered through a $100 \mu \mathrm{m}$ filter (Merck Millipore, Billerica, MA, USA) and pellet resuspended in $0.9 \%$ saline. SVF yield was determined using a Muse FACS cell counter (Merck Millipore) with a nuclear count and cell viability dye (Merck Millipore). An autologous concentration of platelets in a small plasma volume (PRP), having an abundance of growth factors to promote soft and hard tissue healing, was prepared from the case study patients by centrifugation of $3 \times 6 \mathrm{~mL}$ of blood (per knee joint) with anticoagulant citrate dextrose solution - A at $450 \mathrm{~g}$ for 10 minutes.

Posttreatment rehabilitation comprised of a 4-month supervised progressive resistance training program incorporating specific water- and land-based exercises designed to improve physical function, strength, QOL and to reduce pain levels. Supervised rehabilitation sessions were performed twice per week in month 1 , three times per week in month 2 , and four times per week in months 3 and 4 .

The specific exercise program was as follows: month 1, a water-based exercise program using body weight exercises each session, twice weekly; month 2 , the introduction of weekly land-based exercises using body weights combined with additional water-based resistance exercises, twice weekly; month 3, increasing land-based resistance exercises to twice weekly, while continuing with twice weekly waterbased resistance exercises; month 4, resistance training was progressively increased for both the land- and water-based exercises.

Prior to treatment, the patients were administered the Knee Injury and Osteoarthritis Outcome Score (KOOS) questionnaire, and then monthly for 12 months. KOOS is a patient self-reported questionnaire that overcomes observer administration bias. It measures five separately scored subscales or clinical conditions that include Pain; Symptoms; Activities of Daily Living (ADL); Function in Sport and Recreation (Sport/Rec); and knee-related QOL. Functional assessment was also performed monthly over 12 months by the "Get-up and Go" (GUG) test and the "Stair Climbing Test" (SCT) for measures of balance and mobility. ${ }^{21}$ The rate of perceived exertion (RPE) scale (0-10) was used to 
Table I Effect of combinatorial treatment on KOOS subscale scores for Case I

\begin{tabular}{|c|c|c|c|c|c|c|c|c|c|c|c|c|c|c|}
\hline \multirow[t]{3}{*}{ KOOS subscales } & \multicolumn{7}{|l|}{ Left knee } & \multicolumn{7}{|l|}{ Right knee } \\
\hline & \multirow[t]{2}{*}{ Pretreatment } & \multicolumn{6}{|c|}{ Posttreatment (months) } & \multirow[t]{2}{*}{ Pretreatment } & \multicolumn{6}{|c|}{ Posttreatment (months) } \\
\hline & & 2 & 3 & 6 & 8 & 10 & 12 & & 2 & 3 & 6 & 8 & 10 & 12 \\
\hline Pain & 81 & 75 & 100 & 94 & 100 & 100 & 100 & 75 & 64 & 100 & 100 & 100 & 100 & 100 \\
\hline Symptoms & 86 & 86 & 96 & 93 & 96 & 100 & 100 & 79 & 82 & 86 & 86 & 96 & 96 & 96 \\
\hline ADL & 59 & 59 & 100 & 97 & 100 & 100 & 100 & 71 & 65 & 100 & 100 & 100 & 100 & 100 \\
\hline Function in Sport/Rec & 50 & 60 & 100 & 85 & 95 & 95 & 100 & 45 & 35 & 100 & 100 & 100 & 100 & 100 \\
\hline Knee-related QOL & 0 & 0 & 38 & 63 & 81 & 100 & 100 & 31 & 25 & 38 & 100 & 100 & 100 & 100 \\
\hline
\end{tabular}

Notes: Effect of combinatorial treatment on Case I KOOS subscales. Mean KOOS subscale scores for pretreatment and posttreatment 2, 3, 6, 8, I0, and I2 months for Pain, Symptoms, ADL, Function in Sport/Rec, and knee-related QOL. Table data represent transformed raw scale scores to a 0-100 scale, where $0=$ extreme knee problem and $100=$ no knee problem.

Abbreviations: KOOS, Knee Injury and Osteoarthritis Outcome Score; ADL, Activities of Daily Living; Sport/Rec, Sport and Recreation; QOL, Quality of Life.

indicate physical strain, with 10 being the most difficult and 0 being the easiest. ${ }^{22}$

\section{Case I}

A 23-year-old male patient, $100 \mathrm{~kg}$ weight and $1.93 \mathrm{~m}$ height (body mass index [BMI] 26.8) was assessed. Clinical inspection and MRI confirmed osteoarthritis of both knees. Initial KOOS subscale scores for the left knee were recorded as Pain 81; Symptoms 86; ADL 59; Function in Sport/Rec 50, and knee-related QOL 0 . The right knee KOOS subscale scores were Pain 75; Symptoms 79; ADL 71; Function in Sport/Rec 45, and knee-related QOL 31. Pretreatment functional assessment by SCT reported a RPE of 8 out of 10 for both knees, and a GUG score of 8 (left knee) and 0 (right knee).

Following baseline measurements of KOOS, the patient was given a single dose of SVF $\left(7.5 \times 10^{7}\right.$ cells $)$ and PRP per knee. The patient received further injections of PRP into the knees at the $2 \mathrm{nd}, 3 \mathrm{rd}$, and 4 th month. Moderate exercise therapy began at 6 months posttreatment for 4 months, consisting of both water- and land-based exercises. Monthly assessment of the patient's knee and associated problems were recorded by use of the KOOS (Table 1), SCT (Figure 1), and GUG test (Figure 2).

At final follow-up, the patient improved to the maximum of a $100 \mathrm{KOOS}$ score in all five subscales (left knee) and $a \geq 96$ score for the right knee in all five subscales of Pain, Symptoms, ADL, Sport/Rec function, and knee-related QOL. The patient completely regained normal functional activity for both knees as measured by the RPE scale for the SCT (Figure 1), and for the left knee by the GUG test (Figure 2) by 12 months.

\section{Case 2}

A 59-year-old female, $85 \mathrm{~kg}$ weight and $1.65 \mathrm{~m}$ in height (BMI 31.2), was assessed. Clinical examination and MRI confirmed osteoarthritic disorder of both knees, with a pretreatment, functional assessment of 7 for both knees

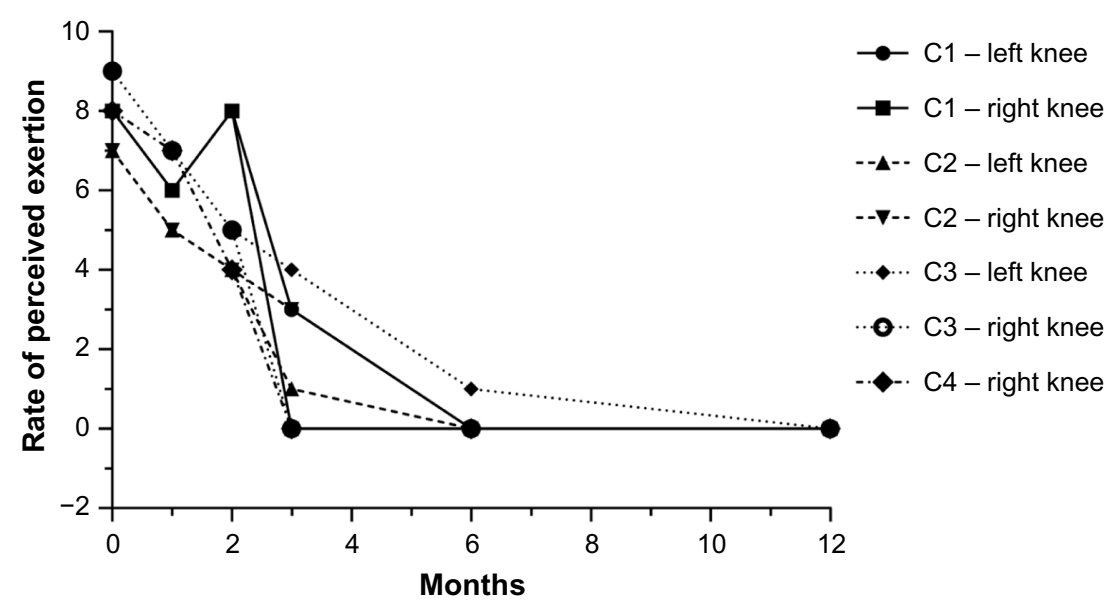

Figure I Functional assessment of the knee by the "Stair Climbing Test" over 12 months.

Notes: A 9-step "Stair Climbing Test" assessed monthly by the ability to ascend and descend a flight of stairs, as well as lower extremity strength, power, and balance. The rate of perceived exertion scale (0-10) was used to indicate physical strain, with 10 being the most difficult and 0 being the easiest. C4 - left knee data was not provided as the knee was normal and untreated.

Abbreviations: $\mathrm{Cl}$, Case I; C2, Case 2; C3, Case 3; C4, Case 4. 


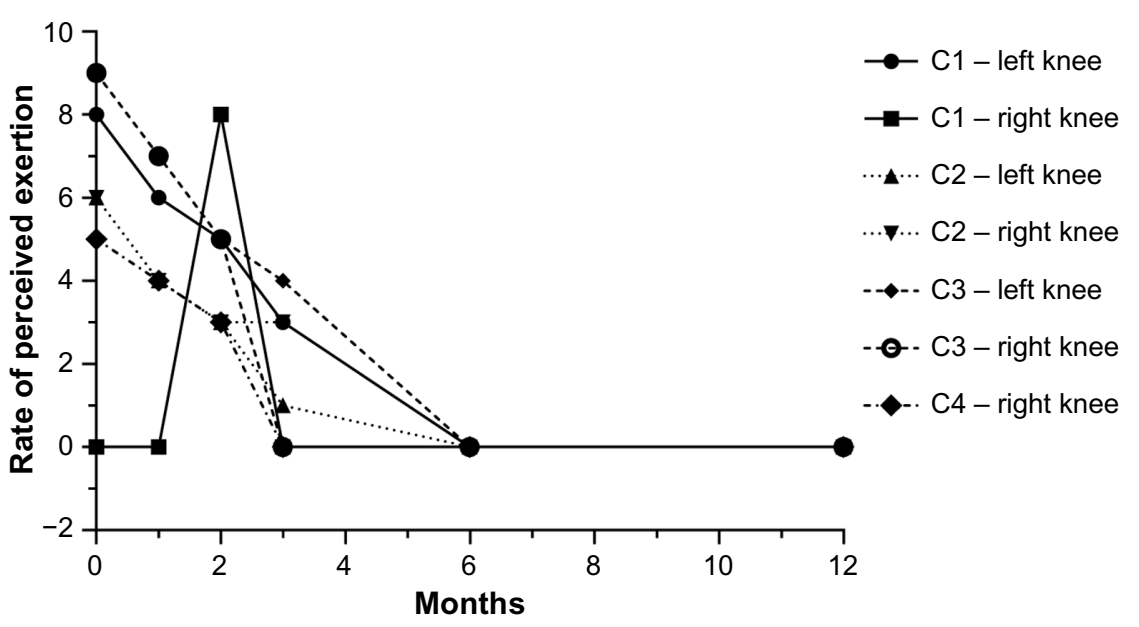

Figure 2 Functional assessment of the knee by the "Get-Up and Go" test over 12 months.

Notes: A "Get-Up and Go" test was assessed monthly by the subject rising from a chair, walking $3 \mathrm{~m}$, turning around, and returning to the chair to sit down. The rate of perceived exertion scale $(0-10)$ was used to indicate physical strain, with 10 being the most difficult and 0 being the easiest. C4 - left knee data was not provided as the knee was normal and untreated.

Abbreviations: $\mathrm{Cl}$, Case I; C2, Case 2; C3, Case 3; C4, Case 4

by the SCT. The GUG test documented a RPE of 6 for both knees. The pretreatment KOOS subscale scores for both knees were Pain 56; Symptoms 25; ADL 74; Function in Sport/ Rec 15, and knee-related QOL 50. The patient was given a single dose of SVF $\left(5.0 \times 10^{7}\right)$ cells in addition to $3 \mathrm{~mL}$ of PRP. The patient received further injections of PRP into the knees at the 2nd, 3rd, and 4th month. Six months posttreatment the patient began an exercise program consisting of water- and land-based exercises. KOOS and functional test scores were monitored monthly to determine the effectiveness of the combined SVF cells, PRP, and moderate exercise therapy. Twelve months posttreatment the left and right knees improved in all five subscales (Table 2). The left knee scored 100 in all subscales, and the right knee improved to Pain 97; Symptoms 86; ADL 100; Sport/Rec function 85, and kneerelated QOL 94. Functional assessment on the RPE by the SCT and GUG test indicated a normal score of 0 for both knees at 12 months (Figures 1 and 2, respectively).

\section{Case 3}

The patient was a 74-year-old male with a body weight of $94 \mathrm{~kg}$ and $1.78 \mathrm{~m}$ in height (BMI 29.7). Clinical assessment showed osteoarthritic disorder of both knees with KOOS subscale scores of Pain 28; Symptoms 43; ADL 34; Function in Sport/Rec 10, and knee-related QOL 13 for the left knee. The right knee KOOS profile was Pain 36; Symptoms 43; ADL 40; Sport/Rec function 10, and a knee-related QOL 19. Initial functional assessment by the SCT recorded a RPE of 9 for both knees and 9 by the GUG test (both knees). Following assessment, the patient was given a single dose of SVF $\left(10.0 \times 10^{7}\right)$ cells obtained from the patient's own lipoaspirate and $3 \mathrm{~mL}$ of autologous PRP. The patient received further injections of PRP into the knees at the 2nd, 3rd, and 4th month. Three months posttreatment the patient began a moderate exercise program consisting of water- and land-based exercises. The KOOS subscale scores for Pain, Symptoms, and ADL improved to $\geq 99$ for both knees (Table 3). The

Table 2 Effect of combinatorial treatment on KOOS subscale scores for Case 2

\begin{tabular}{|c|c|c|c|c|c|c|c|c|c|c|c|c|c|c|}
\hline \multirow[t]{3}{*}{ KOOS subscales } & \multicolumn{7}{|l|}{ Left knee } & \multicolumn{7}{|l|}{ Right knee } \\
\hline & \multirow[t]{2}{*}{ Pretreatment } & \multicolumn{6}{|c|}{ Posttreatment (months) } & \multirow[t]{2}{*}{ Pretreatment } & \multicolumn{6}{|c|}{ Posttreatment (months) } \\
\hline & & 2 & 3 & 6 & 8 & 10 & 12 & & 2 & 3 & 6 & 8 & 10 & 12 \\
\hline Pain & 56 & 67 & 100 & 100 & 100 & 100 & 100 & 56 & 67 & 78 & 100 & 78 & 89 & 97 \\
\hline Symptoms & 25 & 57 & 96 & 100 & 100 & 100 & 100 & 25 & 57 & 54 & 79 & 68 & 75 & 86 \\
\hline ADL & 74 & 76 & 100 & 100 & 100 & 100 & 100 & 74 & 76 & 85 & 99 & 88 & 97 & 100 \\
\hline Function in Sport/Rec & 15 & 35 & 75 & 100 & 100 & 100 & 100 & 15 & 35 & 55 & 75 & 75 & 90 & 85 \\
\hline Knee-related QOL & 50 & 69 & 94 & 100 & 100 & 100 & 100 & 50 & 69 & 69 & 81 & 100 & 94 & 94 \\
\hline
\end{tabular}

Notes: Effect of combinatorial treatment on Case 2 KOOS subscales. Mean KOOS subscale scores for pretreatment and posttreatment 2, 3, 6, 8, I0, and I2 months for Pain, Symptoms, ADL, Function in Sport/Rec, and QOL. Table data represent transformed raw scale scores to a $0-100$ scale, where $0=$ extreme knee problem and $100=$ no knee problem.

Abbreviations: KOOS, Knee Injury and Osteoarthritis Outcome Score; ADL, Activities of Daily Living; Sport/Rec, Sport and Recreation; QOL, Quality of Life. 
Table 3 Effect of combinatorial treatment on KOOS subscale scores for Case 3

\begin{tabular}{|c|c|c|c|c|c|c|c|c|c|c|c|c|c|c|}
\hline \multirow[t]{3}{*}{ KOOS subscales } & \multicolumn{7}{|l|}{ Left knee } & \multicolumn{7}{|l|}{ Right knee } \\
\hline & \multirow[t]{2}{*}{ Pretreatment } & \multicolumn{6}{|c|}{ Posttreatment (months) } & \multirow[t]{2}{*}{ Pretreatment } & \multicolumn{6}{|c|}{ Posttreatment (months) } \\
\hline & & 2 & 3 & 6 & 8 & 10 & 12 & & 2 & 3 & 6 & 8 & 10 & 12 \\
\hline Pain & 28 & 64 & 58 & 89 & 100 & 100 & 100 & 36 & 67 & 56 & 83 & 100 & 100 & 100 \\
\hline Symptoms & 43 & 68 & 68 & 79 & 100 & 100 & 100 & 43 & 68 & 64 & 75 & 96 & 96 & 100 \\
\hline$A D L$ & 34 & 65 & 62 & 87 & 99 & 99 & 99 & 40 & 57 & 53 & 87 & 99 & 99 & 99 \\
\hline Function in Sport/Rec & 10 & 30 & 75 & 50 & 60 & 60 & 60 & 10 & 30 & 45 & 50 & 60 & 60 & 60 \\
\hline Knee-related QOL & 13 & 44 & 63 & 50 & 81 & 81 & 94 & 19 & 44 & 50 & 44 & 81 & 81 & 94 \\
\hline
\end{tabular}

Notes: Effect of combinatorial treatment on Case 3 KOOS subscales. Mean KOOS subscale scores for pretreatment and posttreatment 2, 3, 6, 8, I0, and I2 months for Pain, Symptoms, ADL, Function in Sport/Rec, and QOL. Table data represent transformed raw scale scores to a $0-100$ scale, where $0=$ extreme knee problem and $100=$ no knee problem.

Abbreviations: KOOS, Knee Injury and Osteoarthritis Outcome Score; ADL, Activities of Daily Living; Sport/Rec, Sport and Recreation; QOL, Quality of Life.

Function in Sport/Rec and knee-related QOL improved to 60 and 94, respectively (both knees). The RPE for the SCT and GUG test were determined to be normal $(0)$ at 12 months post combined treatment (Figures 1 and 2, respectively).

\section{Case 4}

A 50-year-old female weighing $60 \mathrm{~kg}$ and $1.60 \mathrm{~m}$ height (BMI 23.4). Clinical evaluation confirmed osteoarthritis of the right knee. Pretreatment KOOS subscale scores of Pain 64; Symptoms 21; ADL 81; Function in Sport/Rec 10, and kneerelated QOL 25 were reported (Table 4). Initial functional assessment by the SCT and GUG test confirmed an RPE of 8 of 5 , respectively for the right knee. The patient was given a single dose of SVF $\left(6.0 \times 10^{7}\right)$ cells and $3 \mathrm{~mL}$ of PRP to the right knee following extraction from the patient's own lipoaspirate. The patient received further injections of PRP into the knee at the 2nd, 3rd, and 4th month. Three months posttreatment the patient began an exercise program consisting of water- and land-based exercises. KOOS scores improved to normal (100) in all five subscales (Table 4). Measures of functional assessments using

Table 4 Effect of combinatorial treatment on KOOS subscale scores for Case 4

\begin{tabular}{lllllllll}
\hline KOOS Subscales & \multicolumn{1}{l}{ Right knee } \\
\cline { 3 - 8 } & Pretreatment & \multicolumn{10}{l}{ Posttreatment } & (months) \\
\cline { 3 - 8 } & & $\mathbf{2}$ & $\mathbf{3}$ & $\mathbf{6}$ & $\mathbf{8}$ & $\mathbf{1 0}$ & $\mathbf{1 2}$ \\
\hline Pain & 64 & 67 & 92 & 92 & 94 & 100 & 100 \\
Symptoms & 21 & 61 & 79 & 96 & 89 & 96 & 100 \\
ADL & 81 & 96 & 97 & 100 & 99 & 100 & 100 \\
Function in Sport/Rec & 10 & 50 & 80 & 100 & 90 & 100 & 100 \\
Knee-related QOL & 25 & 63 & 63 & 94 & 75 & 94 & 100 \\
\hline
\end{tabular}

Notes: Effect of combinatorial treatment on Case 4 KOOS subscales. Mean KOOS subscale scores for pretreatment and posttreatment 2, 3, 6, 8, 10, and 12 months for Pain, Symptoms, ADL, Function in Sport/Rec, and QOL. Table data represents transformed raw scale scores to a $0-100$ scale, where $0=$ extreme knee problem and $100=$ no knee problem.

Abbreviations: KOOS, Knee Injury and Osteoarthritis Outcome Score; ADL, Activities of Daily Living; Sport/Rec, Sport and Recreation; QOL, Quality of Life. the SCT and GUG test indicated a normal exertion score of 0 , (Figures 1 and 2, respectively).

\section{Conclusion}

Osteoarthritis disorders affect the entire joint and result from the degradation of cartilage and subchondral bone in articulated joints. The resulting outcome for patients is pain and inflammation (which may be severe), stiffness, tenderness, and in some cases effusion. ${ }^{23}$ Subsequently, there is diminished functional activity, decreased QOL, increased susceptibility to falls, and increased morbidity and mortality. As there are currently no cures for osteoarthritis, treatment is based on relieving symptoms and improving function.

Of the seven joints treated, all demonstrated an improvement to $\geq 94$ in knee-related QOL. Six joints improved to near normal $(\geq 96)$ for KOOS Pain and Symptoms subscales with three of the joints improving to normal (100) in all five KOOS subscales. All patients demonstrated improvement in all five KOOS subscales of $>8-10$ points (Minimal Important Change) for all joints. ${ }^{24}$ There are a number of limitations with this study, including that the changes observed wholly or partly, may be due to placebo effect. Other confounding factors of the study are the relative importance of each individual component (StroMed, PRP, and exercise) to the clinical improvements, and whether only one or all of the therapies were required to obtain the benefits. It is also not possible with this study to unravel the effect (both shortand long-term) that posttreatment rehabilitation had on the outcome scores, with a large portion of the benefits observed prior to exercise commencement. It could be suggested that SVF/PRP treatment may have improved the long-term inflammatory environment, and that controlled exercise led to additional improvements in pain and activity levels through musculoskeletal readjustment and strengthening, although additional studies are required to explore this hypothesis. 
This case series, while encouraging, requires control clinical trials for combinatorial therapy in the treatment of knee osteoarthritis and an understanding of the structural, clinical, molecular, and cellular outcomes.

This is the first reported case of using ultrasonified lipoaspirate (StroMed) to treat osteoarthritis in a combinatorial approach utilizing PRP and posttreatment rehabilitation exercise. Overall, the data suggest that this approach may be effective in reducing pain and inflammation, and in restoring movements and functional activity for patients with osteoarthritis of the knee. Combinational StroMed therapy also demonstrated improvement in the patients' kneerelated QOL and functional exertion. The use of StroMed may have great potential to delay or even prevent future complex knee replacement surgery with all of its associated complications.

\section{Acknowledgment}

Written informed consent was obtained from the patients for publication of this case report and accompanying images.

\section{Author contributions}

Rod Diamond designed and supervised the exercise program. Nathan Gibbs, Rod Diamond, Eric O Sekyere, and Wayne D Thomas performed the procedures, collected the patient data, and assisted with completion of the manuscript. All authors contributed toward drafting and critically revising the paper, and read and approved the final manuscript.

\section{Disclosure}

The use of ultrasonic cavitation to obtain SVF from adipose is a filed patent by Cell-Innovations Pty Ltd. Wayne Thomas is a director and shareholder of Cell-Innovations Pty Ltd. The authors report no other conflicts of interest in this work.

\section{References}

1. Porrello ER, Mahmoud AI, Simpson E, et al. Transient regenerative potential of the neonatal mouse heart. Science. 2011;331(6020): 1078-1080.

2. Wei X, Yang X, Han ZP, Qu FF, Shao L, Shi YF. Mesenchymal stem cells: a new trend for cell therapy. Acta Pharmacol Sin. 2013;34(6):747-754.

3. Crisan M, Yap S, Casteilla L, et al. A perivascular origin for mesenchymal stem cells in multiple human organs. Cell Stem Cell. 2008;3(3): 301-313.

4. Salgado AJ, Reis RL, Sousa NJ, Gimble JM. Adipose tissue derived stem cells secretome: soluble factors and their roles in regenerative medicine. Curr Stem Cell Res Ther. 2010;5(2):103-110.
5. Schaffler A, Buchler C. Concise review: adipose tissue-derived stromal cells - basic and clinical implications for novel cell-based therapies. Stem Cells. 2007;25(4):818-827.

6. Goel A, Sangwan SS, Siwach RC, Ali AM. Percutaneous bone marrow grafting for the treatment of tibial non-union. Injury. 2005;36(1):203-206.

7. Gangji V, Hauzeur JP, Matos C, De Maertelaer V, Toungouz M, Lambermont M. Treatment of osteonecrosis of the femoral head with implantation of autologous bone-marrow cells. A pilot study. $J$ Bone Joint Surg Am. 2004;86-A(6):1153-1160.

8. Buda R, Vannini F, Cavallo M, et al. One-step arthroscopic technique for the treatment of osteochondral lesions of the knee with bonemarrow-derived cells: three years results. Musculoskelet Surg. 2013; 97(2):145-151.

9. Gan Y, Dai K, Zhang P, Tang T, Zhu Z, Lu J. The clinical use of enriched bone marrow stem cells combined with porous beta-tricalcium phosphate in posterior spinal fusion. Biomaterials. 2008;29(29):3973-3982.

10. Pak J. Regeneration of human bones in hip osteonecrosis and human cartilage in knee osteoarthritis with autologous adipose-tissue-derived stem cells: a case series. J Med Case Rep. 2011;5:296.

11. Zuk PA, Zhu M, Mizuno H, et al. Multilineage cells from human adipose tissue: implications for cell-based therapies. Tissue Eng. 2001;7(2): 211-228.

12. Fraser JK, Wulur I, Alfonso Z, Hedrick MH. Fat tissue: an underappreciated source of stem cells for biotechnology. Trends Biotechnol. 2006;24(4):150-154.

13. Kern S, Eichler H, Stoeve J, Kluter H, Bieback K. Comparative analysis of mesenchymal stem cells from bone marrow, umbilical cord blood, or adipose tissue. Stem Cells. 2006;24(5):1294-1301.

14. Jurgens WJ, Oedayrajsingh-Varma MJ, Helder MN, et al. Effect of tissue-harvesting site on yield of stem cells derived from adipose tissue: implications for cell-based therapies. Cell Tissue Res. 2008; 332(3):415-426

15. Riordan NH, Ichim TE, Min WP, et al. Non-expanded adipose stromal vascular fraction cell therapy for multiple sclerosis. J Transl Med. 2009;7:29.

16. Varma MJ, Breuls RG, Schouten TE, et al. Phenotypical and functional characterization of freshly isolated adipose tissue-derived stem cells. Stem Cells Dev. 2007;16(1):91-104.

17. Ichim TE, Harman RJ, Min WP, et al. Autologous stromal vascular fraction cells: a tool for facilitating tolerance in rheumatic disease. Cell Immunol. 2010;264(1):7-17.

18. Felson DT, Lawrence RC, Dieppe PA, et al. Osteoarthritis: new insights. Part 1: the disease and its risk factors. Ann Intern Med. 2000;133(8):635-646.

19. Patel S, Dhillon MS, Aggarwal S, Marwaha N, Jain A. Treatment with platelet-rich plasma is more effective than placebo for knee osteoarthritis: a prospective, double-blind, randomized trial. Am J Sports Med. 2013;41(2):356-364.

20. Bright R, Bright M, Bright P, Hayne S, Thomas WD. Migraine and tension-type headache treated with stromal vascular fraction: a case series. J Med Case Rep. 2014;8:237.

21. Mathias S, Nayak US, Isaacs B. Balance in elderly patients: the "get-up and go" test. Arch Phys Med Rehabil. 1986;67(6):387-389.

22. Borg GA. Psychophysical bases of perceived exertion. Med Sci Sports Exerc. 1982;14(5):377-381.

23. Bhatia D, Bejarano T, Novo M. Current interventions in the management of knee osteoarthritis. J Pharm Bioallied Sci. 2013;5(1):30-38.

24. Roos EM, Lohmander LS. The Knee Injury and Osteoarthritis Outcome Score (KOOS): from joint injury to osteoarthritis. Health Qual Life Outcomes. 2003;1:64. 
Journal of Pain Research

\section{Publish your work in this journal}

The Journal of Pain Research is an international, peer-reviewed, open access, online journal that welcomes laboratory and clinical findings in the fields of pain research and the prevention and management of pain. Original research, reviews, symposium reports, hypothesis formation and commentaries are all considered for publication. a very quick and fair peer-review system, which is all easy to use. Visit http://www.dovepress.com/testimonials.php to read real quotes from published authors.

\footnotetext{
Submit your manuscript here: http://www.dovepress.com/journal-of-pain-research-journal
} 